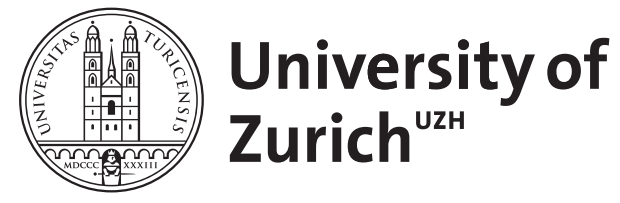

Zurich Open Repository and Archive

University of Zurich

University Library

Strickhofstrasse 39

CH-8057 Zurich

www.zora.uzh.ch

Year: 2017

\title{
Minimally invasive analgesia after cardiac surgery
}

\author{
Cassina, Tiziano
}

DOI: https://doi.org/10.1097/EJA.0000000000000557

Posted at the Zurich Open Repository and Archive, University of Zurich

ZORA URL: https://doi.org/10.5167/uzh-144942

Journal Article

Published Version

Originally published at:

Cassina, Tiziano (2017). Minimally invasive analgesia after cardiac surgery. European Journal of Anaesthesiology, 34(2):54-55.

DOI: https://doi.org/10.1097/EJA.0000000000000557 


\title{
Minimally invasive analgesia after cardiac surgery
}

\author{
Tiziano Cassina
}

European Journal of Anaesthesiology 2017, 34:54-55

This Invited Commentary accompanies the following original article:

Eljezi V, Imhoff E, Bourdeaux D, et al. Bilateral sternal infusion of ropivacaine and length of stay in ICU after cardiac surgery with increased respiratory risk. A randomised controlled trial. Eur J Anaesthesiol 2017;34:56-65

During the pioneer phase of cardiac surgery, postoperative pain was considered a minor clinical adverse effect. At that time, the attention of the cardiac anaesthetist was focused on the risk of postoperative cardiovascular failure rather than on the patient's comfort. The main fear was postoperative myocardial depression induced by ischaemia during aortic clamping and cardioplegic cardiac arrest during extracorporeal circulation. The paradigm proposed was a protective, haemodynamically stable, highdose opioid narcosis. General anaesthesia was based on large amounts of intravenous opioids to induce profound analgesia in response to surgical stimulation. The aim was to avoid hypertension and tachycardia when the surgeon performed sternotomy. The bradycardia produced by the opioids was viewed as a suitable effect in patients with ischaemic heart disease, as beta blockers were not routinely utilised in the perioperative period. However, due to its slow metabolism, large amounts or repeated small doses of fentanyl resulted in higher and longer lasting plasma concentrations. ${ }^{1}$ As a result, the patient took advantage of a progressive smooth awakening after surgery, supported by the ventilator and mechanical ventilation in the ICU. Over time, the number of cardiac surgery patients rapidly increased thereby creating extra pressure on the availability of beds in ICU. As a consequence, the approach had to be changed and a different routine was required.
In the early nineties, the concept of the fast-track pathway in cardiac surgery was introduced. Although more directed at early extubation, this new trend involved a multifactorial approach aimed at improving care in cardiosurgical patients. ${ }^{2}$ Intraoperative high-dose narcotic anaesthesia switched to lighter volatile based anaesthesia, or intravenous short-acting anaesthetic agents, allowing early respiratory weaning. The objective was to end general anaesthesia soon after surgery and restore the patient's respiratory autonomy. Rapid extubation improved the interaction between patient and caregiver, allowing early mobilisation and intensive postoperative respiratory physiotherapy. Therefore, improved pain control after cardiac surgery emerged as a challenge to improve patient comfort and reduce complications. ${ }^{3}$ Unfortunately, pain after cardiac surgery is a multifactorial condition involving median sternotomy, chest opening, internal mammary dissection, mediastinal and pleural drains, and saphenous vein harvest. Continuous intravenous infusion of opioids seemed to be a valid therapy, but it induces several adverse effects, including respiratory depression, decreased gastrointestinal motility, nausea and vomiting. These undesirable complications were deleterious to the patient, potentially leading to an unfavourable outcome. Multimodal analgesia, based on nonopioid systemic analgesics or a combination of different pain control techniques, appeared a valid alternative.

Several analgesic approaches have been investigated in patients undergoing cardiac surgery. In this setting, intrathecal or epidural analgesia, performed before the induction of general anaesthesia, was attempted. Neuroaxial use of local anaesthetics, opioids or both, allowed the control of intraoperative and postoperative pain. ${ }^{4}$ This strategy, associated with short-acting anaesthesia agents, made extubation possible while still in the operation theatre. ${ }^{5}$ Although good analgesic results were demonstrated, the clinical application of neuro-axial analgesia, combined with general anaesthesia, remains problematic in cardiac surgery. The most frequent

From the Cardiac Anaesthesia and Intensive Care Department, Fondazione Cardiocentro Ticino, Lugano, Faculty of Medicine, University Hospital of Geneva Switzerland Correspondence to Prof Tiziano Cassina, MD, Cardiac Anaesthesia and Intensive Care Department, Fondazione Cardiocentro Ticino, Via Tesserete 48, 6900 Lugano, Switzerland

E-mail: tiziano.cassina@cardiocentro.org 
adverse effect is sympathetic nerve blockade that induces vasoplegia and hypotension. Pruritus, nausea and urinary retention are also commonly observed. But the most threatening complication is epidural bleeding in patients who require anticoagulation during cardiopulmonary bypass that may occur in up to $1: 3500$ procedures. Finally, central neuraxial techniques are not routinely performed on patients undergoing cardiac surgery because of procedural risks, dual antiplatelet therapy and the lack of a consensus about the real impact on morbidity and mortality. ${ }^{6}$ Paravertebral local anaesthetic infiltration has not been widely adopted by cardiac anaesthetists or surgeons despite promising results, as median sternotomy needs a bilateral approach. ${ }^{7}$

Continuous wound infusion of local anaesthetics for pain control has been shown to be efficacious for a variety of surgical procedures. ${ }^{8}$ In this issue of the Journal, Eljezi et al. ${ }^{9}$ report the results of a randomised, observer-blinded controlled trial in 120 patients with high respiratory complication risks after cardiac surgery. The authors compared a postoperative systemic intravenous opioid based protocol with subcutaneous analgesia. The primary end-point was the time needed for eligibility for ICU discharge, according to established clinical criteria included in a checklist. Three independent observers blindly assessed the fulfilment of all discharge criteria. The final ICU length of stay analysis showed no significant statistical difference (intent-to-treat $P=0.680$, perprotocol $P=0.873$ ). This result is not so surprising, as postoperative cardiac surgery management is based on a comprehensive multifactorial recovery programme. In this process, pain control represents only one component, among others, that improves patient care. The authors reported enhanced patient comfort in the intervention group $(P<0.0001)$, but subcutaneous analgesia needs future technical improvements, as the results of the present study do not show any significant difference in mean morphine consumption between the two groups (45 vs. $43 \mathrm{mg}$ ). Among the encouraging secondary outcomes reported, the lower rate of pulmonary complications (23\% control vs. $4 \%$ intervention group) is noteworthy in this selected subgroup of patients with a high risk of postoperative respiratory complications. The underlying mechanism is likely to be the reduction of dynamic pain allowing early mobilisation, free respiratory movements, facilitation of efficient coughing and less atelectasis. ${ }^{10}$

In summary, analgesia alone does not allow shortening of ICU stay, but does represent a crucial factor in improving the postoperative course. Given the risk of local anaesthetic toxicity, the authors should be congratulated on investigating this potentially serious adverse effect. They found that the minimal plasma threshold for neurotoxicity was surpassed in $22 \%$ of treated patients. Although there were no clinical consequences, this outcome should not be underestimated and the underlying tissue absorption mechanism needs to be understood properly before promoting more widespread use of this technique. This study has limitations, but it highlights how a subcutaneous approach, combined with systemic intravenous analgesia, reduces dynamic pain and respiratory complications after cardiac surgery. This minimally invasive analgesia modality represents a step towards optimal postoperative pain control with low procedural risks.

\section{Acknowledgements relating to this article}

Assistance with the Commentary: none.

Financial support and sponsorship: none.

Conflicts of interest: none.

Comment from the Editor: this Invited Commentary was checked and accepted by the editors but was not sent for external peer review.

\section{References}

1 Lunn JK, Stanley TH, Eisele J, et al. High dose fentanyl anesthesia for coronary artery surgery: plasma fentanyl concentrations and influence of nitrous oxide on cardiovascular responses. Anesth Analg 1979; 58:390395.

2 Cheng DCH, Karski J, Peniston C, et al. Early tracheal extubation after coronary artery bypass graft surgery reduces costs and improves resource use: a prospective, randomized, controlled trial. Anesthesiology 1996; 85:1300-1310.

3 Gust R, Pecher S, Gust A, et al. Effect of patient-controlled analgesia on pulmonary complications after coronary artery bypass grafting. Crit Care Med 1999; 27:2218-2223.

4 Chaney MA. Intrathecal and epidural anesthesia and analgesia for cardiac surgery. Anesth Analg 2006; 102:45-64.

5 Hemmerling TM, Lê N, Olivier JF, et al. Immediate extubation after aortic valve surgery using high thoracic epidural analgesia or opioid-based analgesia. J Cardiothorac Vasc Anesth 2005; 19:176-181.

6 Svircevic V, Passier MM, Nierich AP, et al. Epidural analgesia for cardiac surgery. Cochrane Database Syst Rev 2013;CD006715.

7 Olivier JF, Bracco D, Nguyen P, et al. A novel approach for pain management in cardiac surgery via median sternotomy: bilateral single-shot paravertebral blocks. Heart Surg Forum 2007; 10:357-362.

8 Liu S, Richman JM, Thirlby RC, et al. Efficacy of continuous wound catheters delivering local anesthetic for postoperative analgesia: a quantitative and qualitative systematic review of randomized controlled trials. J Am Coll Surg 2006; 203:914-932.

9 Eljezi V, Imhoff E, Bourdeaux D, et al. Bilateral sternal infusion of ropivacaine and length of stay in ICU after cardiac surgery with increased respiratory risk: a randomised controlled trial. Eur J Anaesthesiol 2017; 34:56-65.

10 Westerdahl E, Lindmark B, Eriksson T, et al. Deep-breathing exercises reduce atelectasis and improve pulmonary function after coronary artery bypass surgery. Chest $2005 ; 128: 3482-4348$. 